\title{
Periostin serves an important role in the pathogenesis of oral squamous cell carcinoma
}

\author{
YUANYUAN KANG $^{1}$, XUE WANG ${ }^{2}$, YING ZHANG $^{1}$ and YAN SUN ${ }^{1}$ \\ ${ }^{1}$ Department of Emergency and Oral Medicine, School of Stomatology, China Medical University, Shenyang, \\ Liaoning 110001; ${ }^{2}$ Department of Orthodontics, Stomatological Hospital of Shenyang, Shenyang, Liaoning 110002, P.R. China
}

Received August 10, 2017; Accepted July 31, 2018

DOI: $10.3892 / \mathrm{ol} .2018 .9660$

\begin{abstract}
The mechanism underlying OSCC tumorigenesis remains unclear. Periostin is considered to be a prominent oncogene in various solid tumors, although its precise role in OSCC progression remains unknown. In the present study, periostin expression was examined in surgical specimens of OSCC cases, and the results were analyzed for possible correlations with clinical characteristics. In addition, the proliferation and invasiveness of OSCC cells were evaluated following transfection with a Periostin small interfering RNA or an overexpression plasmid. The results revealed that periostin levels were significantly higher in patients with OSCC as compared with those in the controls $(\mathrm{P}<0.05)$. In addition, periostin levels in patients with OSCC were significantly associated with permeation classification. Furthermore, periostin expression was observed to promote the proliferation and invasiveness of OSCC cells. The present results suggest that periostin is significantly involved in the pathogenesis of OSCC.
\end{abstract}

\section{Introduction}

Head and neck squamous cell carcinoma (HNSCC) is one of the most prevalent cancers and a major cause of mortality in patients with cancer worldwide, and 330,000 mortality cases are reported each year as a result of this disease (1). Approximately half of these cases involve oral squamous cell carcinoma (OSCC) (2), which is a highly aggressive head and neck tumor prone to local recurrence and metastasis (3). The development of OSCC is a long-term, multistage and multifactorial process, and numerous regulatory factors are involved in its carcinogenesis (4). However, the detailed molecular pathogenesis of this type of cancer remains unclear.

Correspondence to: Dr Yuanyuan Kang, Department of Emergency and Oral Medicine, School of Stomatology, China Medical University, 117 Nanjing North Street, Shenyang, Liaoning 110001, P.R. China

E-mail: kyandly@sina.com

Key words: oral squamous cell carcinoma, periostin, proliferation, invasion, biomarker
An understanding of the molecular mechanisms underlying OSCC tumorigenesis is necessary to identify tumor-specific biomarkers and therapeutic targets for early diagnosis and treatment, respectively.

Periostin, also known as osteoblast-specific factor 2 (fasciclin I-like), is a secreted matrix $\mathrm{N}$-glycoprotein that contains an $\mathrm{NH}_{2}$-terminal signal peptide sequence, internal homologous repeats, a cysteine-rich domain and hydrophilic C-terminal domain $(5,6)$. Periostin performs an important function in numerous biological processes, including bone development, maturation and remodeling, cardiovascular differentiation, and cutaneous and connective tissue remodeling, as well as in allergic diseases, respiratory diseases and various inflammatory conditions (7). Considering the role of periostin in epithelial-mesenchymal transition (EMT), extracellular matrix (ECM) restructuring and remodeling, research has been focusing on the role of periostin in oncology (8).

Therefore, the present study examined periostin expression and its association with the clinical characteristics of OSCC patients, and investigated the possible underlying mechanisms. The study findings suggested that periostin functions as an oncogene and a potential target for OSCC therapy.

\section{Materials and methods}

Ethics statement. Experiments using human samples were approved by the Ethics Committee of the School of Stomatology, China Medical University (Shenyang, China), and written informed consent was obtained from the donors.

Tissue samples. OSCC specimens were obtained from 12 patients who underwent surgical resection in the Department of Oral and Maxillofacial Surgery at the School of Stomatology, China Medical University, between January 2015 and September 2016. These tumor specimens were immediately frozen and stored at $-80^{\circ} \mathrm{C}$. Another set of 90 OSCC specimens, which were fixed with formalin and embedded in paraffin, and 20 OSCC-adjacent healthy epithelium samples were acquired from the Department of Oral Pathology at the School of Stomatology, China Medical University, between January 2012 and March 2016. The specimens used in immunohistochemistry were derived from archival wax blocks in the Department of pathology at the School of Stomatology, China Medical University. 
The 12 cases used in RT-qPCR were originally from the clinical patients. The number of frozen tissue specimens was relatively less. So two different sets of patients used. Patients who received radiotherapy or chemotherapy were excluded. Clinical data, including sex, age, TNM classification, histological grade, stage, tumor location, were obtained from the medical records of the 90 OSCC patients. The TNM classification was assessed according to the World Health Organization 2010 criteria (9). The tumor stage was based on pathological findings according to the American Joint Committee on Cancer (AJCC) guidelines (10).

Immunohistochemical staining. Immunohistochemical staining was performed to detect the protein localization and expression in paraffin-embedded OSCC specimens and adjacent controls. Paraffin sections were cut at $4 \mathrm{~mm}$ thickness. Briefly, the slides were stained with a rabbit anti-periostin polyclonal antibody (ab14041; Abcam, Cambridge, MA, USA) overnight at $4^{\circ} \mathrm{C}$, which was diluted to $1: 1,000$, followed by staining for $1 \mathrm{~h}$ at room temperature with an anti-rabbit IgG polyclonal antibody (sc515946; Santa Cruz Biotechnology, Inc., Dallas, TX, USA), which was diluted to 1:10,000. Each slide was evaluated by one of the authors under a microscope (Nikon Corporation, Tokyo, Japan). At higher magnification (x400), five visual fields were selected randomly, and the positive expression signals were analyzed by means of the Image-Pro Plus 6.0 software (Media Cybernetics, Inc., Rockville, MD, USA). Periostin protein levels in the OSCC tissue and adjacent healthy epithelium samples were compared in accordance with the integral optical density (IOD) as a parameter for semi-quantitative detection.

Cell culture. Human OSCC cell lines SCC-9 and SCC-25 (ATCC, Manassas, VA, USA) were used between passages 10 and 20. In addition, human keratinocytes (HaCaT cells; ATCC) between passages 4 and 6 were used as the controls. The OSCC cells were maintained in Dulbecco's modified Eagle's medium (DMEM; Gibco; Thermo Fisher Scientific, Inc., Waltham, MA, USA) supplemented with $10 \%$ fetal bovine serum and $1 \%$ of penicillin-streptomycin solution, whereas $\mathrm{HaCaT}$ cells were cultured in DMEM and 10\% fetal bovine serum (Gibco; Thermo Fisher Scientific, Inc.). All the cell lines were maintained at $37^{\circ} \mathrm{C}$ in the presence of $5 \% \mathrm{CO}_{2}$.

Reverse transcription-quantitative polymerase chain reaction (RT-qPCR). Total RNA was extracted from the tissues and cells with the TRIzol reagent (Sangon Biotech Co., Ltd., Shanghai, China). Following quantification of RNA concentration using an ultraviolet spectrophotometer, $1 \mu \mathrm{g}$ DNase-treated RNA was used for cDNA synthesis with an RT reagent kit (Takara Biotechnology Co., Ltd., Dalian, China). The RT reaction was conducted at $37^{\circ} \mathrm{C}$ for $15 \mathrm{~min}$ and $85^{\circ} \mathrm{C}$ for $5 \mathrm{~min}$, and the RT reaction system was as follows: $1 \mu \mathrm{g}$ RNA and $4 \mu 15 \mathrm{X}$ PrimeScript RT Master Mix, with RNase-free distilled $\mathrm{H}_{2} \mathrm{O}$ added up to $20 \mu \mathrm{l}$. Next, qPCR was conducted by means of the SYBR Premix Ex Taq II kit (Takara Biotechnology Co., Ltd.) with the following reaction system: $10 \mu \mathrm{l}$ SYBR Premix Ex Taq II, $1 \mu \mathrm{l}$ cDNA, $0.5 \mu \mathrm{l}$ forward primer, $0.5 \mu 1$ reverse primer and $8 \mu \mathrm{l}$ sterile water. The cycling conditions were as follows: $95^{\circ} \mathrm{C}$ for $1 \mathrm{~min}, 94^{\circ} \mathrm{C}$ for $30 \mathrm{sec}, 58^{\circ} \mathrm{C}$ for $30 \mathrm{sec}$ and $72^{\circ} \mathrm{C}$ for $10 \mathrm{sec}$. The sequences of the primer pairs were as follows: Periostin, 5'-TTTACAACGGGCAAATACTGG AAAC-3' (forward) and 5'-GATGATCTCGCGGAATATGTG AA-3' (reverse); GAPDH, 5'-ACCACAGTCCATGCCATC AC-3' (forward) and 5'-TCCACCACCCTGTTGCTGTA-3' (reverse). Gene expression was normalized to $G A P D H$ as the internal control, and the mean relative change was determined in triplicate or quintuplicate through relative quantification and application of the $2^{-\Delta \Delta \mathrm{Cq}}$ method (11).

Western blotting. OSCC cells were washed three times with cold PBS, and then with a lysis buffer containing $1 \mathrm{mM}$ PMSF (Beyotime Institute of Biotechnology Shanghai, China). The tissue samples were collected and sonicated in a lysis buffer containing $1 \mathrm{mM}$ PMSF. Protein concentrations were determined by the bicinchoninic acid method. Next, samples corresponding to $50 \mu \mathrm{g}$ total protein were subjected to SDS-PAGE in a $10 \%$ gel, and then transferred onto polyvinylidene difluoride membranes. Subsequent to blocking with $5 \%$ non-fat milk for $1 \mathrm{~h}$ at room temperature, the membranes were incubated with anti-periostin (1:1,000, ab14041; Abcam, Cambridge, MA, USA) at $4^{\circ} \mathrm{C}$ overnight. Following washing with TBST three times at room temperature, each time for $10 \mathrm{~min}$, they were incubated with horseradish peroxidase conjugated rabbit anti-mouse secondary antibodies (1:10,000; ab6728; Abcam, Cambridge, MA, USA) for $1 \mathrm{~h}$ and then were washed again Protein bands were visualized with an enhanced chemiluminescence reagent (Pierce; Thermo Fisher Scientific, Inc.). GAPDH (1:10,000, ab8245; Abcam) was used as the internal control. Image J 1.44 software (National Institutes of Health, Bethesda, MD, USA) was used for the analysis of Periostin expression.

Plasmid construction and siRNA synthesis. The open reading frame of human periostin cDNA was cloned into eukaryotic expression vector GV144 (GeneChem, Shanghai, China). Subsequently, the amplicon of the periostin gene was purified, digested and ligated into the respective HindIII and EcoRI sites of the GV144 vector. The Periostin overexpression plasmid sequences as follows: 5'-GTCCGGACTCAGATC TCGAGCTATGATTCCCTTTTTACCCATG-3' (forward) and 5'-TATCTAGATCCGGTGGATCCTCACTGAGAACGA CCTTCCCTTAATC-3' (reverse). A GV144 empty vector was used as control for Periostin expression. The siPeriostin and siCtrl were synthesized by GenePharam company (Shanghai, China). The siPeriostin sequence was as follows: 5'-GCCAUC ACAUCGGACAUAUTT-3' (forward) and 5'-AUAUGUCCG AUGUGAUGGCTT-3' (reverse). The siCtrl sequence was as follows: 5'-UUCUCCGAACGUGUCACGUTT-3' (forward) and 5'-ACGUGACACGUUCGGAGAATT-3' (reverse).

Transfection with POSTN small interfering RNA (siRNA) and overexpression plasmid. OSCC cells were routinely cultured. OSCC cells were transfected with the siPeriostin for Periostin expression knockdown and with the periostin plasmid for Periostin overexpression, using Lipofectamine 2000 (Thermo Fisher Scientific, Inc.) according to the manufacturer's protocol. The siPeriostin sequence was as follows: 5'-CCC AUGGAGAGCCAAUUAUTT-3'. An empty vector was 
Table I. IOD of periostin in OSCC by immunohistochemical staining.

\begin{tabular}{lc}
\hline Tissue & IOD \\
\hline OSCC & $156.02 \pm 7.12^{\mathrm{a}}$ \\
Adjacent & $63.23 \pm 8.15$ \\
\hline
\end{tabular}

${ }^{\mathrm{a}} \mathrm{P}<0.05$ vs. adjacent tissue. The median \pm interquartile values are shown. IOD, integral optical density; OSCC, oral squamous cell carcinoma.

transfected into the control group. The transfection efficiency was then assessed by western blotting and RT-qPCR analyses.

MTS cell proliferation assay. OSCC cells were transfected with siPeriostin or with the periostin plasmid for $24 \mathrm{~h}$ at $37^{\circ} \mathrm{C}$. Proliferation assays were conducted using an MTS Cell Proliferation Assay kit (Promega Corp., Madison, WI, USA). Briefly, cells with a stable knockdown of periostin expression or control cells were plated in 96-well plates at a density of $2 \times 10^{3}$ cells/well.

The culture plates were taken out at different time periods $(24,48$, and $72 \mathrm{~h})$ and continuously cultured at $37^{\circ} \mathrm{C}$ for $2 \mathrm{~h}$ after the addition of $10 \mu 1$ MTS in each well. The optical density (OD) value was measured at a wavelength of $490 \mathrm{~nm}$ using enzyme linked immunosorbent assay.

Cell invasion assays. OSCC cells were transfected with siPeriostin or with the periostin plasmid for $24 \mathrm{~h}$. Invasion assays were then performed using a Cell Invasion Assay kit (BD Biosciences, Billerica, MA, USA). Six-well plates and transwell chambers was used. The upper chamber was pre-coated with $50 \mu \mathrm{l}$ of $20 \%$ growth factor-reduced Matrigel for the invasion assay. Briefly, cells were plated in the upper well of a Boyden chamber at a concentration of $5 \times 10^{4}$ cells $/$ well in $100 \mu 1$ serum-free DMEM. In the lower chamber, $600 \mu \mathrm{l}$ DMEM containing $10 \%$ FBS was added to serve as a chemoattractant. After incubation at $37^{\circ} \mathrm{C}$ for $20 \mathrm{~h}$, the invading cells on the lower surface of the filter were fixed with $95 \%$ ethanol and stained with the Coomassie blue dye (Leagene Biotechnology Co., Ltd., Beijing, China). Subsequently, images of the cells were captured, and the cell numbers were manually counted in five random visual fields per filter (magnification, $\mathrm{x} 40$ ).

Statistical analysis. The Mann-Whitney rank sum test was applied to compare the periostin mRNA and protein levels between the OSCC cells and the healthy controls. In addition, correlations between the IOD levels of the periostin protein and clinical factors were assessed by univariate analysis. Statistical analysis was performed using the SPSS version 17.0 software (SPSS, Inc., Chicago, IL, USA). P $<0.05$ was considered to indicate a statistically significant difference.

\section{Results}

High expression of periostin in OSCC tissues and cell lines. First, the expression of periostin in OSCC and adjacent tissues,
A
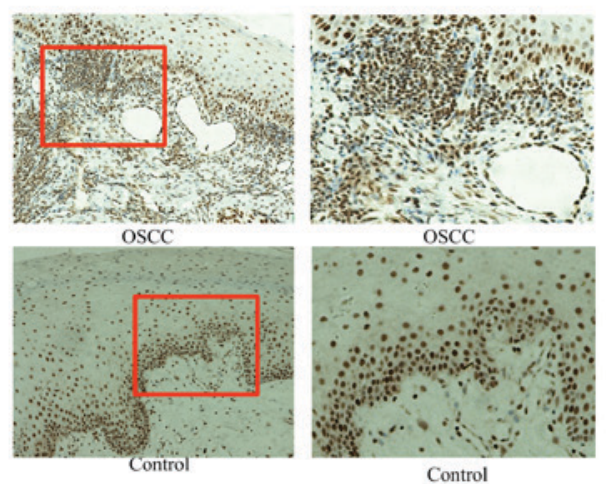

B

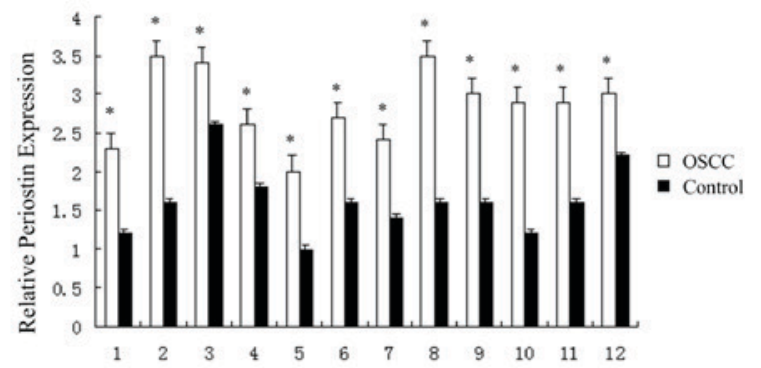

C

D
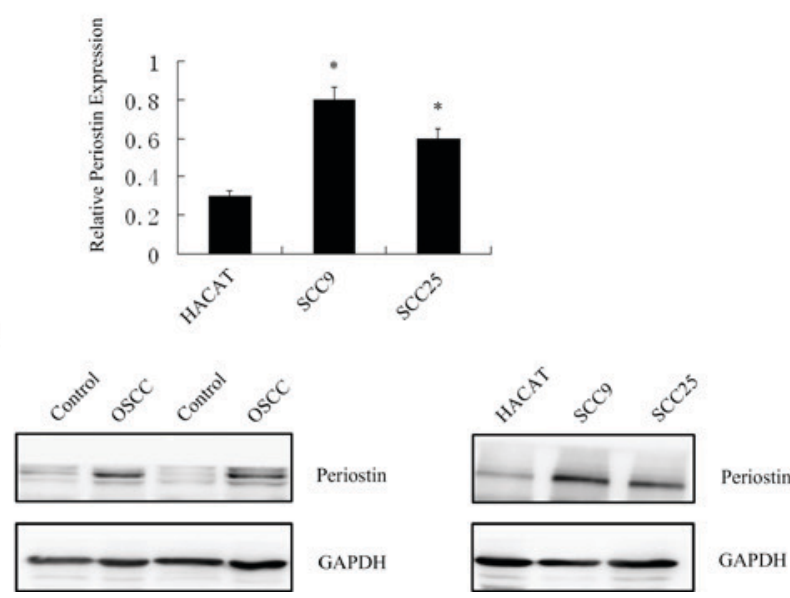

Figure 1. Periostin expression in OSCC. (A) Representative images of periostin immunohistochemical staining (x200) and cells in the red boxes at $\mathrm{x} 400$, and (B) mRNA expression of periostin examined by RT-qPCR in OSCC and healthy adjacent tissues. (C) Expression of periostin mRNA was examined by RT-qPCR in OSCC cells. (D) Protein expression of periostin was evaluated by western blotting in OSCC tissues and cell lines. ${ }^{*} \mathrm{P}<0.05$ vs. corresponding control group. OSCC, oral squamous cell carcinoma; RT-qPCR, reverse transcription-quantitative polymerase chain reaction.

including 110 cases (90 OSCC and 20 healthy adjacent tissues), were examined by immunohistochemistry (Fig. 1A). Periostin staining was mainly detected in the cytoplasm and was occasionally present in the nuclei of tumor cells. The OSCC group exhibited significantly higher periostin expression as compared with that in the adjacent tissue controls (Table I; $\mathrm{P}<0.05)$.

To determine the expression levels of periostin, the specimens of OSCC and healthy adjacent tissues were collected and RT-qPCR and western blotting were then conducted. 
Table II. Association of the clinical characteristics of oral squamous cell carcinoma patients with positive $(\mathrm{n}=78 ; 86.7 \%)$ and negative $(\mathrm{n}=12 ; 13.3 \%)$ periostin expression.

\begin{tabular}{|c|c|c|c|c|}
\hline Characteristic & $\mathrm{N}$ & Periostin positive, $\mathrm{n}(\%)$ & Periostin negative, n (\%) & P-value \\
\hline Sex & & & & 0.571 \\
\hline Male & 55 & $50(64.1)$ & $9(75.0)$ & \\
\hline Female & 35 & $28(35.9)$ & $3(25.0)$ & \\
\hline Age (years) & & & & 0.512 \\
\hline$\leq 55$ & 49 & $32(41.0)$ & $6(50.0)$ & \\
\hline$>55$ & 41 & $46(59.0)$ & $6(50.0)$ & \\
\hline $\mathrm{T}$ classification & & & & 0.623 \\
\hline $\mathrm{T} 1 / 2$ & 40 & $30(38.5)$ & $3(25.0)$ & \\
\hline $\mathrm{T} 3 / 4$ & 50 & $48(61.5)$ & $9(75.0)$ & \\
\hline $\mathrm{N}$ classification & & & & 0.704 \\
\hline No & 35 & $47(60.3)$ & $5(41.7)$ & \\
\hline $\mathrm{N} 0+$ & 55 & $31(39.7)$ & $7(58.3)$ & \\
\hline Histological grade & & & & 0.312 \\
\hline Well differentiated & 42 & 49 (62.8) & $6(50.0)$ & \\
\hline Poorly/moderately differentiated & 48 & $29(37.2)$ & $6(50.0)$ & \\
\hline Stage & & & & 0.235 \\
\hline I and II & 36 & $51(65.4)$ & $5(41.7)$ & \\
\hline III and IV & 54 & $27(34.6)$ & $7(58.3)$ & \\
\hline Location & & & & 0.412 \\
\hline Buccal mucosa & 20 & $16(20.5)$ & $4(33.3)$ & \\
\hline Tongue & 45 & $40(51.3)$ & $5(41.7)$ & \\
\hline Mouth floor & 10 & $8(10.3)$ & $2(16.7)$ & \\
\hline Others & 15 & $14(17.9)$ & $1(8.3)$ & \\
\hline Lymphovascular permeation & & & & 0.007 \\
\hline Present & 82 & $72(92.3)$ & $10(83.3)$ & \\
\hline Absent & 8 & $6(7.7)$ & $2(16.7)$ & \\
\hline Distant metastasis & & & & 0.053 \\
\hline Present & 55 & 45 (57.7) & $10(83.3)$ & \\
\hline Absent & 35 & $33(42.3)$ & $2(16.7)$ & \\
\hline
\end{tabular}

For statistical analysis with SPSS software, the data were presented as a fold change in the gene expression, normalized to GAPDH. As shown in Fig. 1B and C, the mRNA $(\mathrm{P}<0.05)$ and protein levels of periostin in OSCC tissue were notably higher in comparison with those in the healthy adjacent tissue. Only 12 sets of samples were shown, as they were the fresh specimens. The 90 cases used in immunohistochemistry were derived from archival wax blocks and are not suitable for RT-qPCR.

RT-qPCR and western blotting were also performed in SCC9, SCC25 and HaCaT cells to measure the expression of periostin. Similar to the tissue results, the mRNA and protein expression levels of periostin were significantly higher in OSCC cells as compared with those in the healthy epithelium cells $(\mathrm{P}<0.05$; Fig. $1 \mathrm{C}$ and $\mathrm{D})$.

Association between periostin and clinical characteristics. The association of periostin expression and the clinical characteristics of OSCC patients was also analyzed in the present study. Univariate analysis was conducted to evaluate any possible associations (Table II). The expression was considered negative if the final IOD was $\geq 65$ and were otherwise considered positive. There was a significant correlation between $\mathrm{N}$ classification and periostin protein levels $(\mathrm{P}=0.007)$. A positive periostin expression was significantly associated with the presence of lymphovascular permeation $(\mathrm{P}=0.007)$. $\mathrm{N}$ classification exhibited borderline significance of correlation $(\mathrm{P}=0.053)$. Other factors were not found to be associated with periostin expression.

Periostin promotes the proliferation and invasiveness of OSCC cells. To determine the physiological role of periostin, the impact of periostin on the growth and motility characteristics of OSCC cells transfected with siPeriostin or the control plasmid was analyzed in SCC9 and SCC25 cell lines. The results revealed that periostin mRNA and protein levels in the two cell lines transfected with negative control were 4-fold higher in comparison with those in cells transfected 

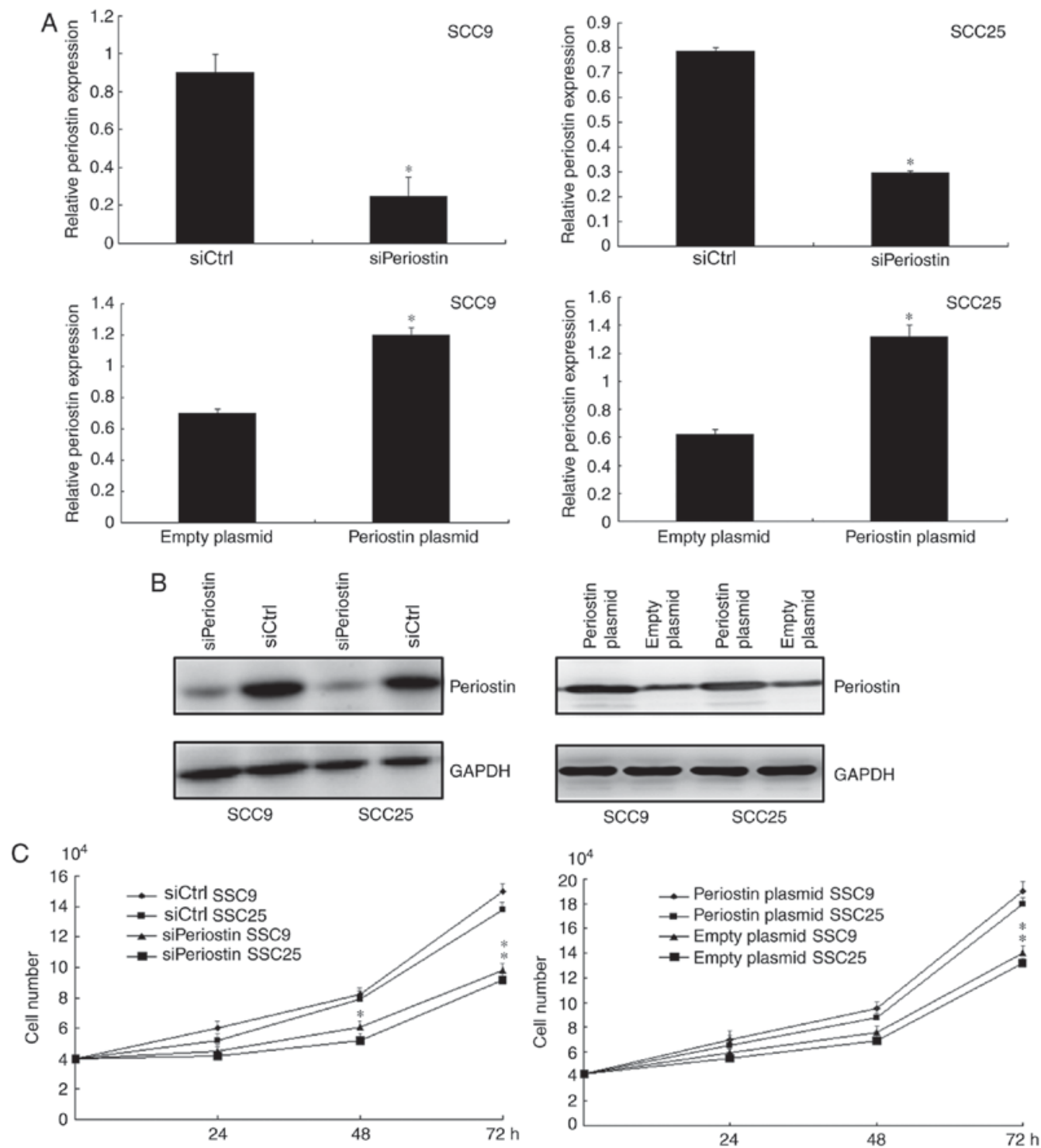

Figure 2. Impact of periostin on the growth characteristics of OSCC cells. (A) mRNA and (B) protein levels of periostin were examined by reverse transcriptionquantitative polymerase chain reaction and western blotting, respectively, after the cells were transfected with the overexpression plasmid or periostin siRNA. (C) Stimulatory effect of periostin on the proliferation of transfected SCC 9 and SCC 25 cells, as evaluated by the MTS assay. ${ }^{*} \mathrm{P}<0.05$ vs. corresponding control group. OSCC, oral squamous cell carcinoma; siRNA, small interfering RNA; Ctrl, control.

with siPeriostin according to the RT-qPCR and western blotting data $(\mathrm{P}<0.05$; Fig. $2 \mathrm{~A}$ and $\mathrm{B})$. In addition, periostin mRNA and protein levels in cells transfected with the periostin plasmid were 2-fold higher compared with those of cells transfected with the empty vector, as determined by RT-qPCR and western blotting $(\mathrm{P}<0.05$; Fig. $2 \mathrm{~A}$ and $\mathrm{B})$. These indicated that transfection with siPeriostin and plasmid successfully resulted in the knockdown and overexpression of periostin, respectively.

To analyze the participation of periostin in the growth of OSCC cells, MTS cell proliferation assays were conducted subsequent to transfection. The results demonstrated that the knockdown of periostin by siPeriostin significantly suppressed the proliferation of OSCC cells, whereas the upregulation of periostin markedly increased the proliferation of OSCC cells (Fig. 2C).

In addition, to examine the potential effect of periostin on the migration of OSCC cells, Transwell assays were conducted in a Boyden chamber. The results revealed that the knockdown of periostin significantly suppressed the invasiveness of OSCC cells, whereas periostin upregulation significantly increased the invasion by OSCC cells (Fig. 3).

\section{Discussion}

Periostin, an extracellular-matrix protein that belongs to the fasciclin family, is encoded by the POSTN gene and has been demonstrated to be essential for the process of remodeling during tissue and organ development or repair, as well as inflammation $(12,13)$. It contains an $\mathrm{N}$-terminal secretory signal peptide, followed by a cysteine-rich domain, four internal homologous repeats and a $\mathrm{C}$-terminal hydrophilic domain. Periostin exerts its biological activities by functioning as a matricellular protein, affecting cell activation by binding to receptors on the cell surface. It participates in numerous biological processes, including the regulation of gene expression and the development 

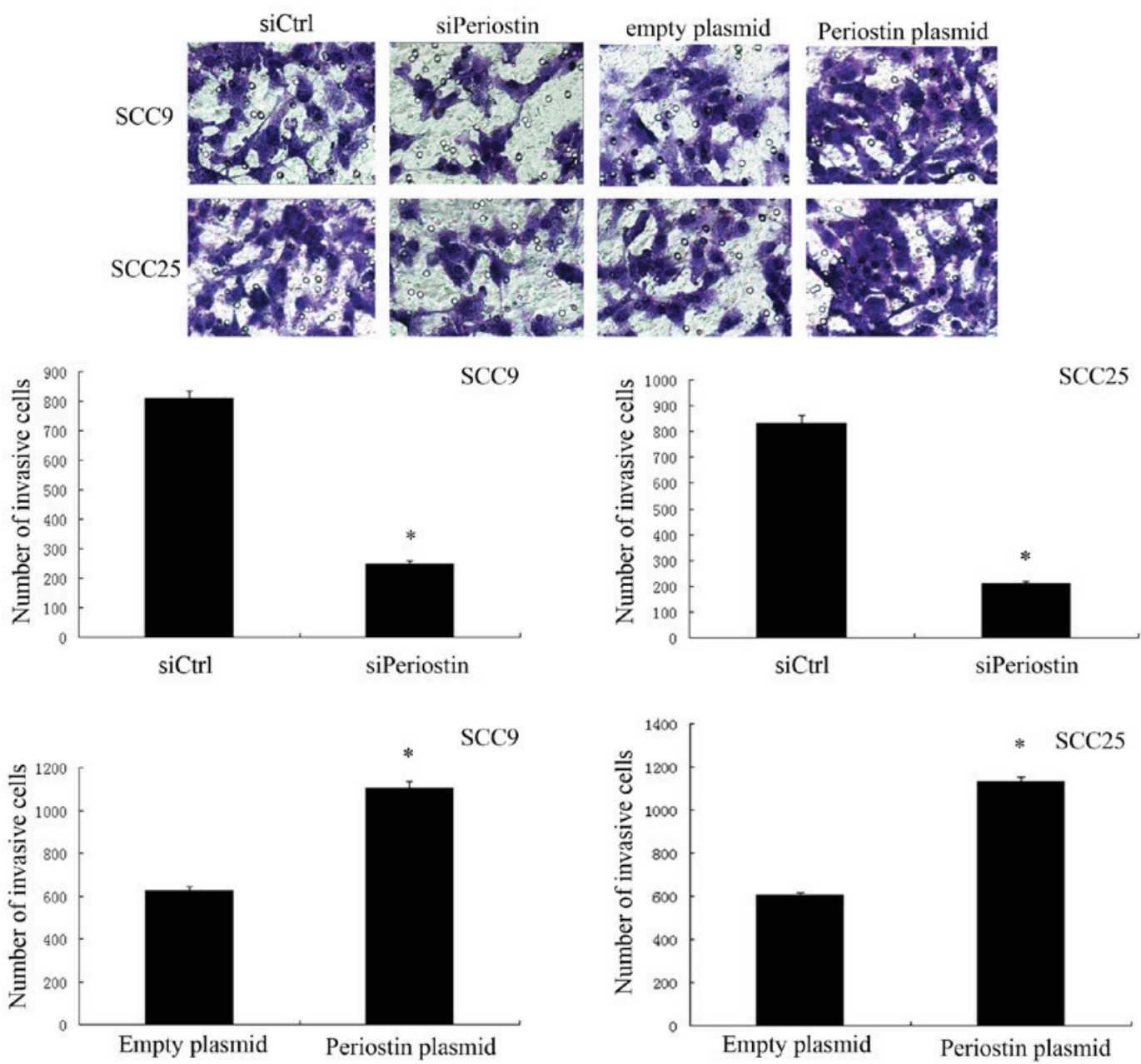

Figure 3. Stimulatory effect of periostin on the invasiveness of SCC9 and SCC25 cells was evaluated by a Transwell assay. ${ }^{*}<0.05$ vs. corresponding control group. si, small interfering RNA; Ctrl, control.

of bone, teeth and heart vessels $(14,15)$. Periostin upregulation has been reported in a number of solid tumors, including colon $(6,16)$, thyroid $(17)$, breast $(18)$, pancreatic $(8,19)$ and lung cancer (20). In addition, accumulating evidence revealed that periostin was upregulated in HNSCC tissue $(21,22)$, and that it promotes invasion and angiogenesis in OSCC (22). Certain studies have also reported that periostin promoted tube formation of lymphatic endothelial cells independently of vascular endothelial growth factor $\mathrm{C}$ and promoted lymphangiogenesis, which was mediated by Src and Akt activity in HNSCC (23). However, the influence of periostin on OSCC progression and metastasis is currently poorly understood.

In the present study, periostin levels were significantly higher in OSCC cell lines as compared with those in $\mathrm{HaCaT}$ cells, which was contrary to the findings of a previous study (21). Furthermore, the present study reported that the knockdown of periostin expression inhibited cell proliferation and invasiveness.

Cases of periostin overexpression were found to be associated with lymphovascular permeation, while distant metastasis exhibited borderline significance of correlation. Consequently, analysis of POSTN expression may be particularly useful during the prognostic evaluation of this cohort of patients.

Although the clinical value of periostin in OSCC was comprehensively analyzed, there are certain limitations in the present study. First, the sample size in the subgroup analysis was not sufficiently large; thus, the statistical power of the results is limited. In addition, the relevant mechanisms were identified in the current study. In our future studies, more in-depth research and analysis will be conducted. Furthermore, animal experiments need to be devised to investigate the effects of periostin on tumor growth in vitro and in vivo.

In conclusion, it was observed that the levels of periostin were highly upregulated in the tumor tissues of patients with OSCC, and immunohistochemical staining demonstrated increased localization to areas of active fibrosis in the tumor tissue. The present study provides a new line of evidence indicating that periostin is significantly upregulated in OSCC tissues and cell lines. The data implied that periostin, as an oncogene, serves an important role in the pathogenesis of OSCC.

\section{Acknowledgements}

Not applicable.

\section{Funding}

This study was supported by grants from the Natural Science Foundation of Liaoning Province (no. 20170541012) and the 
Youth Research Fund of the school of Stomatology, China Medical University (no. K101593-16-05).

\section{Availability of data and materials}

All data generated or analyzed during this study are included in this published article.

\section{Authors' contributions}

YK and XW conceived and designed the experiments. YZ and YS collected and analyzed the imaging and pathology data. YK wrote the manuscript.

\section{Ethics approval and consent to participate}

Experiments using human samples were approved by the Ethics Committee of the School of Stomatology, China Medical University (Shenyang, China), and written informed consent was obtained from the donors.

\section{Patient consent for publication}

Written informed consent was obtained from the donors for the publication of any data and associated images.

\section{Competing interests}

The authors declare that they have no competing interests.

\section{References}

1. Karahatay S, Thomas K, Koybasi S, Senkal CE, Elojeimy S, Liu X, Bielawski J, Day TA, Gillespie MB, Sinha D, et al: Clinical relevance of ceramide metabolism in the pathogenesis of human head and neck squamous cell carcinoma(HNSCC): Attenuation of C(18)-ceramide in HNSCC tumors correlates with lymphovascular invasion and nodal metastasis. Cancer Lett 256: 101-111, 2007.

2. Wang Q, Gao P, Wang X and Duan Y: The early diagnosis and monitoring of squamous cell carcinoma via saliva metabolomics. Sci Rep 4: 6802, 2014.

3. Lo WY, Tsai MH, Tsai Y, Hua CH, Tsai FJ, Huang SY, Tsai $\mathrm{CH}$ and Lai CC: Identification of over-expressed proteins in oral squamous cell carcinoma (OSCC) patients by clinical proteomic analysis. Clin Chim Acta 376: 101-107, 2007.

4. Dong Y, Zhao Q, Ma X, Ma G, Liu C, Chen Z, Yu L, Liu X, Zhang Y, Shao S, et al: Establishment of a new OSCC cell line derived from OLK and identification of malignant transformation-related proteins by differential proteomics approach. Sci Rep 5: 12668, 2015.

5. Huang Y, Liu W, Xiao H, Maitikabili A, Lin Q, Wu T, Huang Z, Liu F, Luo Q and Ouyang G: Matricellular protein periostin contributes to hepatic inflammation and fibrosis. Am J Pathol 185: 786-797, 2015.

6. Li Z, Zhang X, Yang Y, Yang S, Dong Z, Du L, Wang L and Wang C: Periostin expression and its prognostic value for colorectal cancer. Int J Mol Sci 16: 12108-12118, 2015.

7. Oh HJ, Bae JM, Wen XY, Cho NY, Kim JH and Kang GH: Overexpression of POSTN in tumor stroma is a poor prognostic indicator of colorectal cancer. J Pathol Transl Med 51: 306-313, 2017.
8. Baril P, Gangeswaran R, Mahon PC, Caulee K, Kocher HM, Harade T, Zhu M, Kalthoff H, Crnogorac-Jurcevic T and Lemoine NR: Periostin promotes invasiveness and resistance of pancreatic cancer cells to hypoxia-induced cell death: Role of the beta4 integrin and the PI3k pathway. Oncogene 26: 2082-2094, 2007.

9. Campisi G, Giovannelli L, Calvino F, Matranga D, Colella G, Di Liberto C, Capra G, Leao JC, Lo Muzio L, Capogreco M and D'Angelo M: HPV infection in relation to OSCC histological grading and TNM stage. Evaluation by traditional statistics and fuzzy logic model. Oral Oncol 42: 638-645, 2006.

10. O'Connell JB, Maggard MA and Ko CY: Colon cancer survival rates with the new American Joint Committee on Cancer sixth edition staging. J Natl Cancer Inst 96: 1420-1425, 2004.

11. Livak KJ and Schmittgen TD: Analysis of relative gene expression data using real-time quantitative PCR and the 2(-Delta Delta C(T)) method. Methods 25: 402-408, 2001.

12. Koh SJ, Choi Y, Kim BG, Lee KL, Kim DW, Kim JH, Kim JW and Kim JS: Matricellular protein periostin mediates intestinal inflammation through the activation of nuclear factor $\kappa \mathrm{B}$ signaling. PLoS One 11: e0149652, 2016.

13. Rios H, Koushik SV, Wang H, Wang J, Zhou HM, Lindsley A, Rogers R, Chen Z, Maeda M, Kruzynska-Frejtag A, et al: Periostin null mice exhibit dwarfism, incisor enamel defects, and an early-onset periodontal disease-like phenotype. Mol Cell Biol 25: 11131-11144, 2005.

14. Snider P, Hinton RB, Moreno-Rodriguez RA, Wang J, Rogers R, Lindsley A, Li F, Ingram DA, Menick D, Field L, et al: Periostin is required for maturation and extracellular matrix stabilization of noncardiomyocyte lineages of the heart. Circ Res 102: 752-760, 2008.

15. Elliott CG, Wang J, Guo X, Xu SW, Eastwood M, Guan J, Leask A, Conway SJ and Hamilton DW: Periostin modulates myofibroblast differentiation during full-thickness cutaneous wound repair. J Cell Sci 125: 121-132, 2012.

16. Xiao ZM, Wang XY and Wang AM: Periostin induces chemoresistance in colon cancer cells through activation of the PI3K/Akt/survivin pathway. Biotechnol Appl Biochem 62: 401-406, 2015.

17. Fluge $\varnothing$, Bruland O, Akslen LA, Lillehaug JR and Varhaug JE: Gene expression in poorly differentiated papillary thyroid carcinomas. Thyroid 16: 161-175, 2006.

18. Zhang Y, Zhang G, Li J, Tao Q and Tang W: The expression analysis of periostin in human breast cancer. J Surg Res 160: 102-106, 2010.

19. Liu Y and Du L: Role of pancreatic stellate cells and periostin in pancreatic cancer progression. Tumour Biol 36: 3171-3177, 2015.

20. Xu CH, Wang W, Lin Y, Qian LH, Zhang XW, Wang QB and Yu LK: Diagnostic and prognostic value of serum periostin in patients with non-small cell lung cancer. Oncotarget 8: 18746-18753, 2017.

21. Gonzalez HE, Guirati M, Frederick M, Henderson Y, Arumugam J, Spring PW, Mitsudo K, Kim HW and Clayman GL: Identification of 9 genes differentially expressed in head and neck squamous cell carcinoma. Arch Otolaryngol Head Neck Surg 129: 754-759, 2003.

22. Siriwardena BS, Kudo Y, Oqawa I, Kitagawa M, Kitajima S, Hatano H, Tilakaratne WM, Miyauchi M and Takata T: Periostin is frequently overexpressed and enhances invasion and angiogenesis in oral cancer. Br J Cancer 95: 1396-1403, 2006.

23. Kudo Y, lizuka S, Yoshida M, Nguyen PT, Siriwardena SB, Tsunematsu T, Ohbayashi M, Ando T, Hatakeyama D, Shibata T, et al: Periostin directly and indirectly promotes tumor lymphangiogenesis of head and neck cancer. PLoS One 7: e44488, 2012. 